\title{
Subwavelength-grating metamaterial integrated devices for the near- and mid-infrared wavelengths
}

\author{
Alejandro Sánchez-Postigo*a, J. Gonzalo Wangüemert-Pérez ${ }^{\mathrm{a}}$, Alejandro Ortega-Moñux ${ }^{\mathrm{a}}$, \\ Robert Halir ${ }^{\mathrm{a}}$, Daniel Pereira-Martín ${ }^{\mathrm{a}}$, José Manuel Luque-González ${ }^{\mathrm{a}}$, Jens H. Schmid ${ }^{\mathrm{b}}$, \\ Shurui Wang ${ }^{\mathrm{b}}$, Martin Vachon ${ }^{\mathrm{b}}$, Dan-Xia Xu ${ }^{\mathrm{b}}$, Jordi Soler Penadés ${ }^{\mathrm{c}, \mathrm{d}}$, Milos Nedeljkovic ${ }^{\mathrm{c}}$, \\ Goran Z. Mashanovich ${ }^{\mathrm{c}}$, Pavel Cheben ${ }^{\mathrm{b}}$, Íñigo Molina-Fernández ${ }^{\mathrm{a}}$ \\ a'Universidad de Málaga, Dpto. Ingeniería de Comunicaciones, ETSI Telecomunicación, 29071

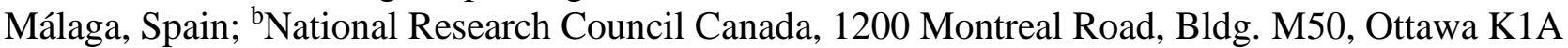 \\ 0R6, Canada; ' Optoelectronics Research Centre, University of Southampton, Southampton, SO17 \\ 1BJ, United Kingdom; ' VLC Photonics S.L., Valencia, Spain
}

\begin{abstract}
The subwavelength patterning of planar structures is now widely used in silicon photonics, enabling the synthesis of metamaterials with engineered optical properties, including refractive index, dispersion, and anisotropy. A wide range of integrated devices based on subwavelength grating (SWG) metamaterials have been demonstrated at telecom wavelengths, some with unprecedented performance. The benefits of SWG metamaterials can be leveraged not only in the typical telecom near-infrared bands, but also at the longer mid-infrared wavelengths. In this invited presentation, we will review our latest developments in SWG-based silicon and germanium photonic devices for the near- and the midinfrared.
\end{abstract}

Keywords: Silicon photonics, Integrated optics, Subwavelength gratings, Metamaterials, Near-infrared, Mid-infrared, Waveguides, Off-chip couplers

\section{INTRODUCTION}

Since their early demonstration at NRC Canada ${ }^{1-7}$, subwavelength grating (SWG) metamaterials in silicon waveguides have been advantageously used to control the flow of light in integrated photonic devices ${ }^{8}$. The refractive index, wavelength dispersion, birefringence, and anisotropy of nanophotonic waveguides can be controlled by judiciously choosing the geometrical parameters of the lithographically defined patterns. This enables the synthesis of metamaterials on-chip, with engineered optical properties. At the near-infrared wavelengths of the $1.55-\mu \mathrm{m}$ telecom band, integrated optical components based on SWG metamaterials — including waveguides, beam splitters, multimode interference couplers, polarization splitters, Bragg filters, and fiber-chip couplers - have been demonstrated with unprecedented performance ${ }^{9}$.

The benefits of SWG metamaterial engineering can also be harnessed at longer wavelengths. The mid-infrared band, comprising the wavelength range of $2-15 \mu \mathrm{m}$, holds promise for important emerging applications, particularly in sensing and free-space optical communications ${ }^{10}$. While silicon-on-insulator (SOI) is becoming established as a leading platform for photonic integration, conventional silicon waveguides have prohibitive loss beyond $\lambda \sim 4 \mu \mathrm{m}$, due to the strong absorption of the silicon dioxide cladding at these longer wavelengths ${ }^{11}$. Various alternatives have been proposed using materials with a wider transparency range (e.g., sapphire, germanium on silicon, etc. ${ }^{12-14}$. We have developed all-silicon and all-germanium SWG metamaterial waveguide platforms with the operational range extending into the midinfrared $^{15-18}$.

Efficient and broadband fiber-chip coupling of light is one of the most significant challenges in silicon photonics ${ }^{19}$. This issue is due to the mode size mismatch between optical fibers (with a typical diameter of $\sim 10 \mu \mathrm{m}$ ) and integrated photonic wires (cross-section of $\sim 500 \mathrm{~nm} \times 220 \mathrm{~nm}$ at $1550-\mathrm{nm}$ wavelength). To cope with the fiber-chip coupling

*asp@ic.uma.es; phone (+34) 952132853 
problem, edge couplers and surface grating couplers are the most used devices, with the latter offering important advantages such as wafer-scale testing and robust misalignment tolerance ${ }^{20}$. A surface grating coupler is a waveguide with a (quasi) periodic perturbation that converts the guided mode into a free-propagating plane wave ${ }^{21}$. The radiated field can be collected by an optical fiber that is positioned above the chip. The coupling efficiency (the fraction of input power that is coupled into the optical fiber) and the 1-dB bandwidth (the range of wavelengths for which the coupling efficiency is $\sim 80 \%$ of its peak value) are the two important parameters in surface grating couplers ${ }^{22}$. State-of-the-art surface grating couplers have a coupling efficiency-bandwidth product of $\sim 60 \mathrm{~nm}$ and incorporate sophisticated techniques such as multilayering ${ }^{24,25}$ and plasmonics ${ }^{26}$, or use non-conventional optical fibers with reduced mode field diameters ${ }^{25,26}$.

In this work, we will show some of our latest advances in silicon photonic components using SWG metamaterials for both the near- and mid-infrared wavelengths, with the emphasis on off-chip couplers. Specifically, we will present a broadband and highly efficient grating coupler using subwavelength grating metamaterials for the near-infrared and a micro-antenna for a novel mid-infrared suspended germanium platform with subwavelength-grating lateral cladding.

\section{ZERO-ORDER GRATING COUPLER FOR THE NEAR-INFRARED BAND}

A zero-order grating coupler is illustrated in Fig. 1. It comprises a periodic waveguide of length $L$, with a subwavelength period $\Lambda$ and a duty cycle $\mathrm{DC}=a / \Lambda$, and a silicon prism with an angle $\phi_{\text {prism }}$ between the input and output surfaces. The prism is tilted at $\phi_{\text {clad }}$ degrees and has an antireflective coating on its output surface. A linear taper of length $L_{\text {taper }}$ is used to adiabatically increase the mode size of the interconnecting waveguide. Then, a fork-shaped transition of length $L_{\text {fork }}$ adapts this mode to the Floquet mode of the grating.

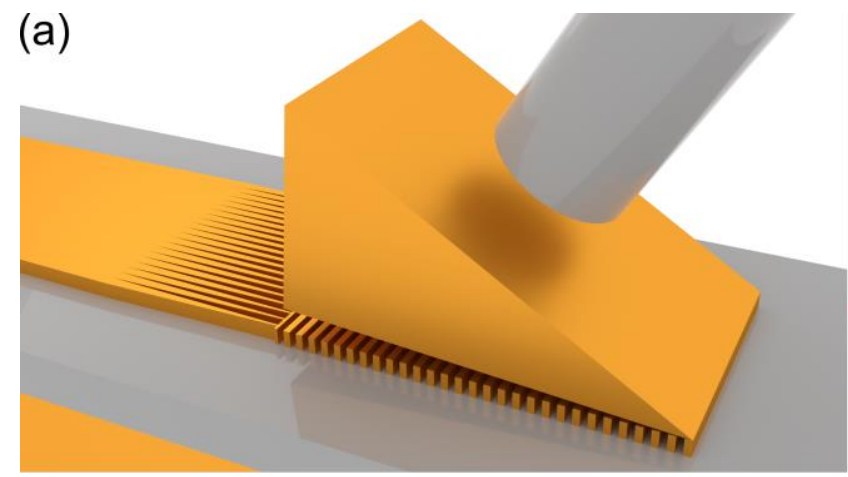

(c)

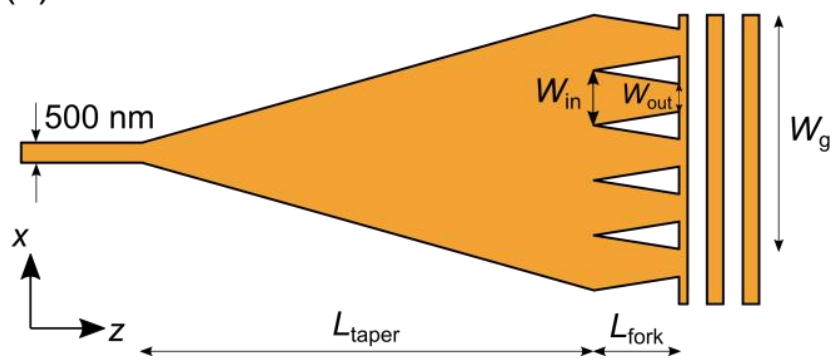

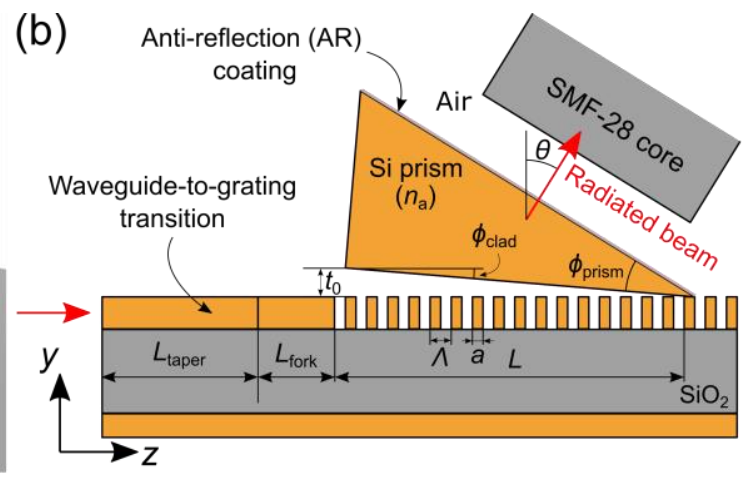

(d)

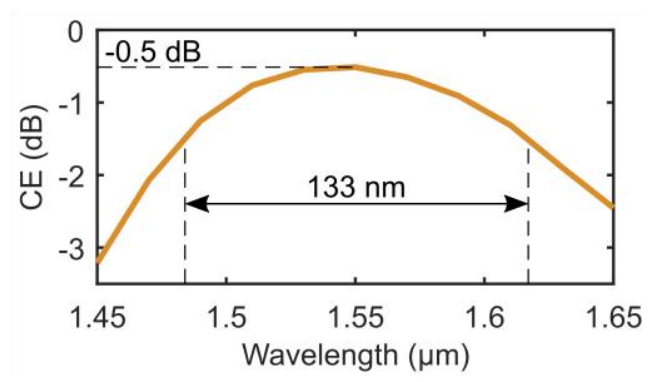

Figure 1. (a) Schematic of a zero-order grating coupler, including the homogeneous-to-SWG injection stage and the core of the single-mode optical fiber. (b) Schematic of a zero-order grating coupler (side view). (c) Schematic of the homogeneousto-SWG injection stage (top view). (d) 3D-FDTD simulated coupling efficiency as a function of the wavelength.

The radiation angle $\theta$ in surface grating couplers is determined by ${ }^{21}$

$$
\theta=\sin ^{-1}\left(\frac{n_{\text {eff }}}{n_{\mathrm{a}}}+m \frac{\lambda}{n_{\mathrm{a}} \Lambda}\right),
$$


where $n_{\text {eff }}$ is the effective index of the Floquet mode supported by the grating waveguide, $n_{\mathrm{a}}$ is the refractive index of the superstrate, $m$ is the diffraction order, $\lambda$ is the operating wavelength, and $\Lambda$ is the grating period. In conventional grating couplers, Eq. (1) is satisfied for $m=-1 \operatorname{order}^{21}$. The explicit dependence of the radiation angle on the wavelength is the primary cause of narrow bandwidth in surface grating couplers. This bandwidth limitation can be overcome by imposing $m=0$, such that Eq. (1) becomes the zero-order grating coupler equation:

$$
\theta=\sin ^{-1}\left(\frac{n_{\text {eff }}}{n_{\mathrm{a}}}\right) .
$$

To enable radiation in the zeroth order, i.e., via evanescent coupling, the period $\Lambda$ is reduced to operate in the subwavelength regime $\left(\Lambda<0.5 \lambda / n_{\text {eff }}\right)$ and a high-refractive-index material is used as a superstrate $\left(n_{\mathrm{a}}>n_{\text {eff }}\right)$. Under these conditions, the bandwidth fundamentally depends on the SWG and material dispersions of $n_{\text {eff }}$ and $n_{\mathrm{a}}$, respectively. To maximize the bandwidth, we choose the geometry that minimizes the SWG dispersion by operating as far as possible from the Bragg regime, i.e., DC $=50 \%$ and $\Lambda=2 \times$ MFS, where MFS is the minimum feature size of the fabrication process. In our case, $\Lambda=200 \mathrm{~nm}$. The coupling efficiency is optimized by tilting the silicon prism $\left(L=20 \mu \mathrm{m}, \phi_{\text {prism }}=\right.$ $35.7^{\circ}, \phi_{\text {clad }}=1^{\circ}$ ). According to our 3D-FDTD simulations, a peak coupling efficiency of $-0.5 \mathrm{~dB}$ and a 1 -dB bandwidth of $133 \mathrm{~nm}$ are achieved [see Fig. 1(d)]. The grating width $W_{\mathrm{g}}=15 \mu \mathrm{m}$ maximizes the overlap with the optical fiber mode in the $x$ direction. A detailed description of our design flow can be found in our theoretical paper ${ }^{23}$. The structural parameters of the input injection stage are $L_{\text {taper }}=200 \mu \mathrm{m}, L_{\text {fork }}=3 \mu \mathrm{m}, W_{\text {in }}=0.5 \mu \mathrm{m}$, and $W_{\text {out }}=0.3 \mu \mathrm{m}$.

The surface grating couplers were fabricated by electron beam lithography and inductively coupled (ICP) high-density plasma etching on an SOI wafer with $220-\mathrm{nm} \mathrm{Si}$ and $3-\mu \mathrm{m} \mathrm{SiO}$ layers. A silicon prism with a base of $2 \mathrm{~mm} \times 2 \mathrm{~mm}$ was suspended on top of the subwavelength grating using a holder and a micro-positioning stage with tilting capabilities. An SEM image of the fabricated coupler is shown in Fig. 2(a).

The coupling efficiency and the bandwidth were measured using two complementary experiments. First, light was coupled in the chip via diffractive surface grating couplers and out of the chip via the zero-order grating coupler under test. The bandwidth of the measured spectral response, $\sim 30 \mathrm{~nm}$, coincides with that of the input conventional grating couplers, confirming the broad bandwidth of the zero-order grating coupler. By subtracting out the loss of the characterization setup and the propagation losses of the waveguides, this experiment provided an upper bound for the coupling efficiency of $-1.0 \mathrm{~dB}$. Subsequently, the bandwidth was measured by injecting light into the chip through the chip facet, which does not limit the spectral response. We obtained a 1-dB bandwidth of $94 \mathrm{~nm}$. A photograph of the facet-to-prism-coupling experimental setup is shown in Fig. 2(b).

Our zero-order grating coupler can be lithographically patterned using standard fabrication processes in microelectronics and has a demonstrated coupling efficiency-bandwidth product of $\sim 75 \mathrm{~nm}$, the highest value yet reported in the literature for surface coupling to an SMF-28 optical fiber. We believe these results open new venues for the development of highperformance surface grating couplers.
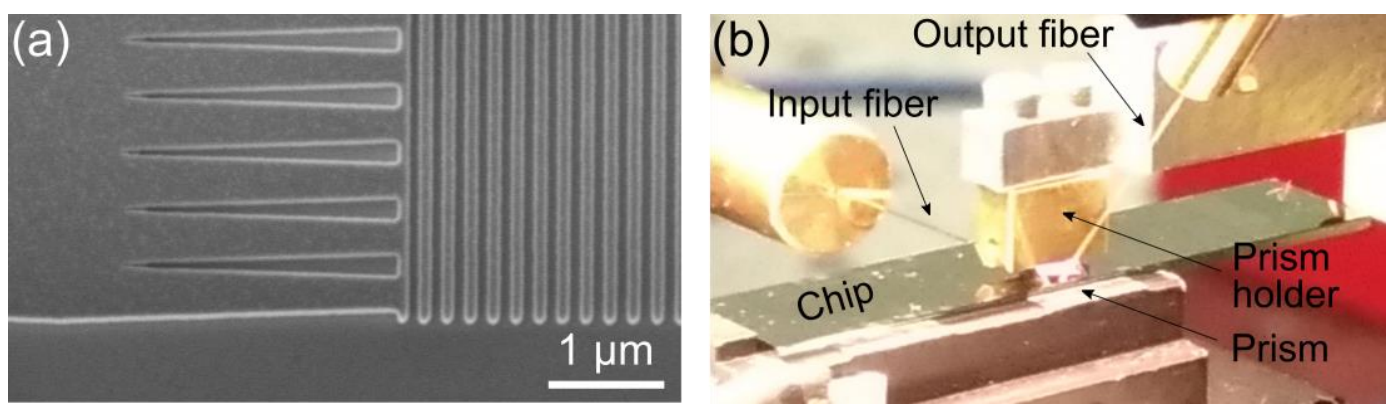

Figure 2. (a) SEM image of the beginning of a zero-order grating couplers, showing the input waveguide-to-grating stage and the first microns of the subwavelength grating. (b) Photograph of the characterization setup that was used to measure the 1 -dB bandwidth of the zero-order grating coupler. 


\section{SUSPENDED GERMANIUM MICRO-ANTENNA FOR THE MID-INFRARED BAND}

Here we present a broadband surface coupler for the suspended germanium platform with subwavelength grating lateral cladding for the mid-infrared wavelength range at a central wavelength of $7.67 \mu \mathrm{m}$ (see Fig. 3) ${ }^{27}$. This germanium platform is based on Ge-on-SOI wafers with thinned Si layer. Suspended waveguides comprise a germanium core that is anchored to lateral germanium slabs by a periodic cladding operating in the subwavelength regime (width $W_{\text {clad }}$, period $\Lambda_{\mathrm{SWG}}$, duty cycle $\mathrm{DC}=a_{\mathrm{SWG}} / \Lambda_{\mathrm{SWG}}$ ). The lateral cladding has a three-fold function: (i) to allow the flow of an HF etching solution that removes the $\mathrm{SiO}_{2}$ bottom layer of the original Ge-on-SOI wafer, (ii) to hold the suspended structures, and (iii) to provide the lateral mode confinement that is required for light guiding. As illustrated in Fig. 3, the proposed suspended germanium fiber-chip surface coupler is formed by three suspended grating periods of pitch $\Lambda$ and duty cycle $\mathrm{DC}=a / \Lambda$ and an adaptation section with a hole and a germainum strip of lengths $b_{\mathrm{ad}}$ and $a_{\mathrm{ad}}$, respectively.

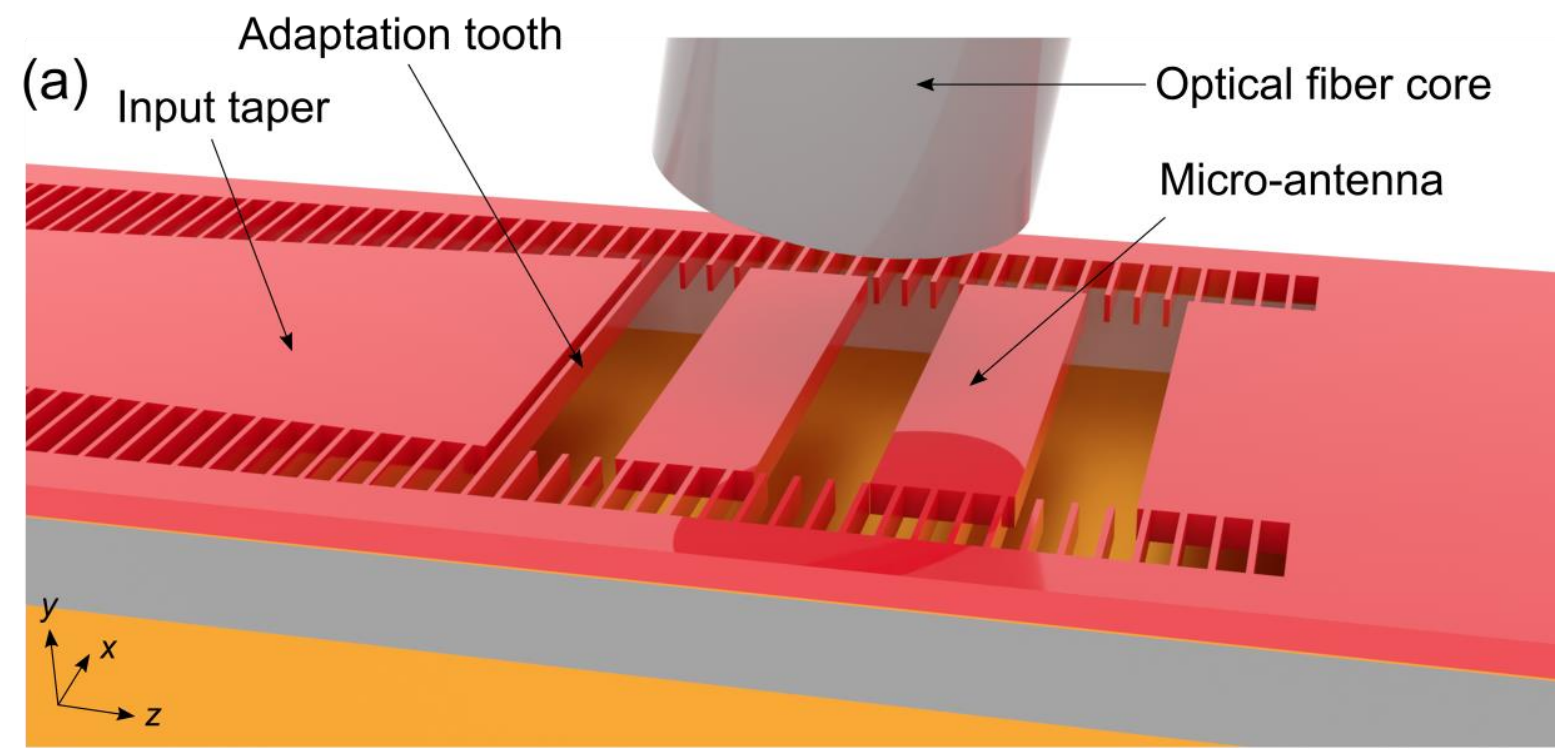

(b)

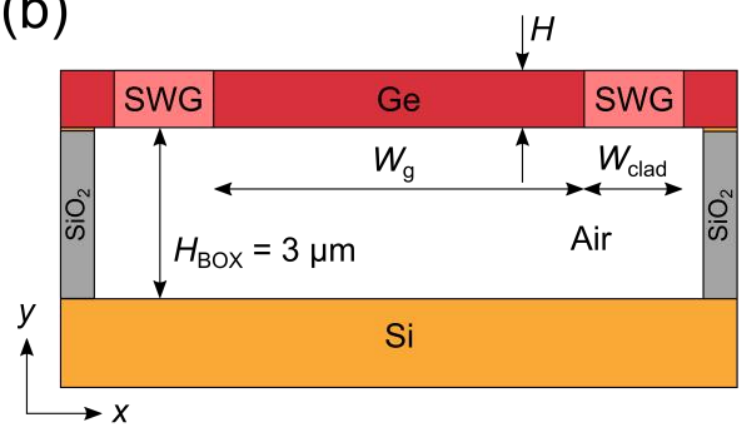

(c)

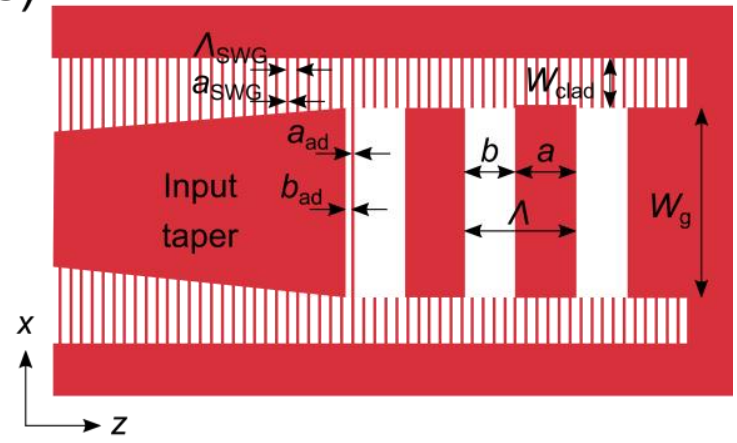

Figure 3. Schematics of the micro-antenna-type fiber-chip coupler for the SWG-cladding suspended germanium platform. (a) 3D view, including the optical fiber core and the input suspended taper. (b) Front view. (c) Top view, including the input taper. The micro-antenna comprises only three radiative elements, with a total length that is comparable to the operating wavelength.

Since the Ge-air index contrast is higher than that in silicon waveguides $\left(n_{\mathrm{Ge}} \sim 4>n_{\mathrm{Si}} \sim 3.5\right)$, the photonic bandgaps of a germanium grating are wider and the range of periods that enable radiation but operate far away from the Bragg regime is smaller ${ }^{28}$. As a result, the design space of broadband and tolerant surface grating couplers is reduced compared to conventional designs in the near-infrared band. Thus, instead of using $m=-1$ in Eq. (1), we relax the typical single-beam design constraint and allow radiation in no more than two diffraction orders simultaneously, provided that only one order carries most of the power. The two-beam condition is satisfied if ${ }^{27}$ 


$$
\Lambda<\frac{\lambda}{n_{\mathrm{a}}},
$$

where $n_{\mathrm{a}}$ is the refractive index of the upper medium (air).

At long-wave infrared wavelengths $(\lambda=7-15 \mu \mathrm{m})$, commercial chalcogenide optical fibers have high numerical aperture, which leads to mode field diameters (MFDs) that are small relative to the wavelength. For example, an SMF-28 optical fiber at $1.55 \mu \mathrm{m}^{29}$ has MFD/ $\lambda=10.4 / 1.55=6.7$, while an IRFlex's IRF-Se-12 at $7.7 \mu \mathrm{m}^{30}$ has MFD/ $\lambda=13.6 / 7.7$ $=1.8$. Consequently, surface grating couplers in the long-wave infrared should operate as optical micro-antennas with much stronger radiation than conventional grating couplers in the near-infrared band.

Using our in-house eigenmode expansion simulator $\left(\right.$ FEXEN $\left.^{31}\right)$, we choose the best design with high coupling efficiency to an IRF-Se-12 optical fiber, radiation angle $<20^{\circ}$ and high tolerance to fabrication imperfections $(\Lambda=7.6 \mathrm{um}, \mathrm{DC}=$ $55 \%)$. Then, we reduce back-reflections by adapting the fundamental mode of input waveguide to that of the microantenna waveguide $\left(a_{\mathrm{ad}}=200 \mathrm{~nm}, b_{\mathrm{ad}}=400 \mathrm{~nm}\right)$. Further details on our design methodology can be found in our previous theoretical work ${ }^{27}$. Using only three radiative elements, we achieve 3D-FDTD simulated coupling efficiency of $-4 \mathrm{~dB}$ and 1-dB bandwidth of $436 \mathrm{~nm}$ [see Fig. 4(a)]. The latter is a fractional bandwidth (BW/ $/$ ) of $\sim 6 \%$, around twice the fractional bandwidth of surface grating couplers in the near-infrared band. Furthermore, a high tolerance to fiber tilt misalignments, or angular bandwidth, of $\pm 10^{\circ}$ is reported [see Fig. 4(c)], which is almost a three-fold improvement over conventional silicon grating couplers at $1550-\mathrm{nm}$ telecom wavelengths. In addition, 2D FDTD simulations reveal that the design is tolerant to fabrication errors of $\pm 150 \mathrm{~nm}$. Figure 4(b) shows an electric field propagation for the TE polarization and $\lambda=7.67 \mathrm{um}$. An SEM image of a suspended germanium micro-antenna, including the input subwavelength-grating waveguide and taper, is shown in Fig. 4(d). To provide mechanical robustness, the lateral cladding of the micro-antenna was not etched, which does not affect the field propagation due to the short length of the structure. While the coupling efficiency has not yet been experimentally corroborated, we measured an angular bandwidth of, at least, $15^{\circ}$. These results constitute a new route for the development of high-performance fiber-chip surface couplers at long-wave infrared wavelengths.

(a)

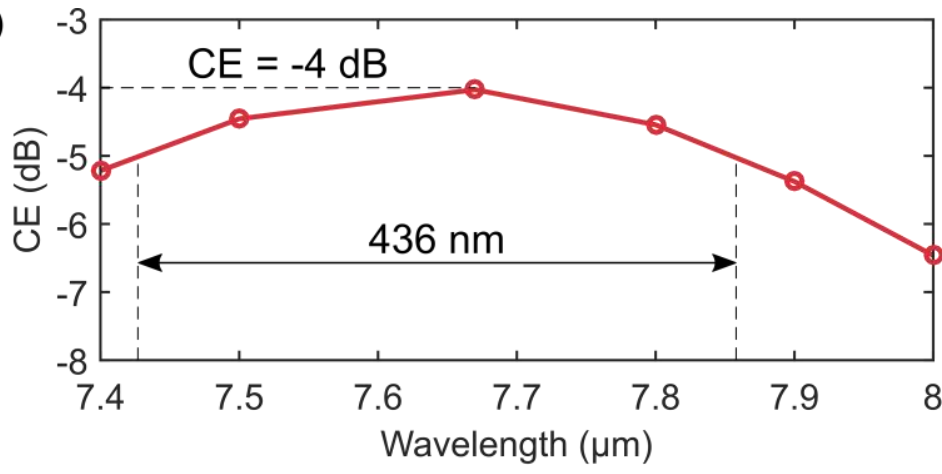

(c)

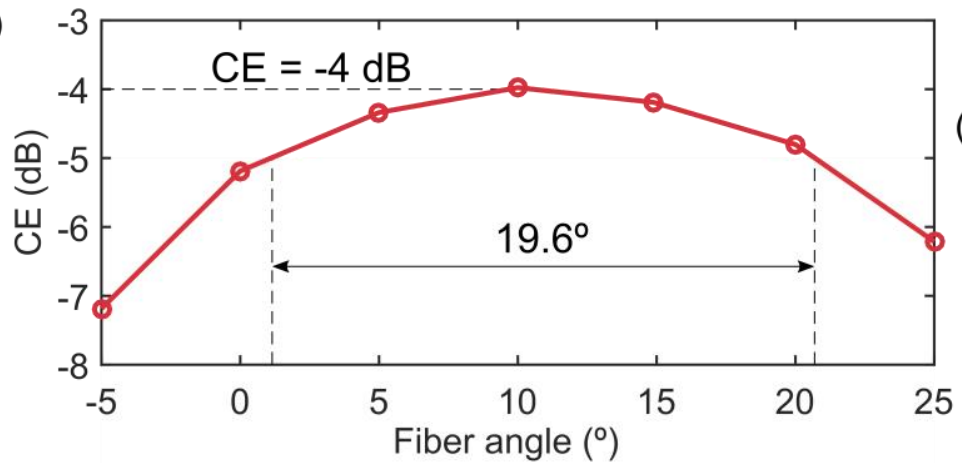

(b)

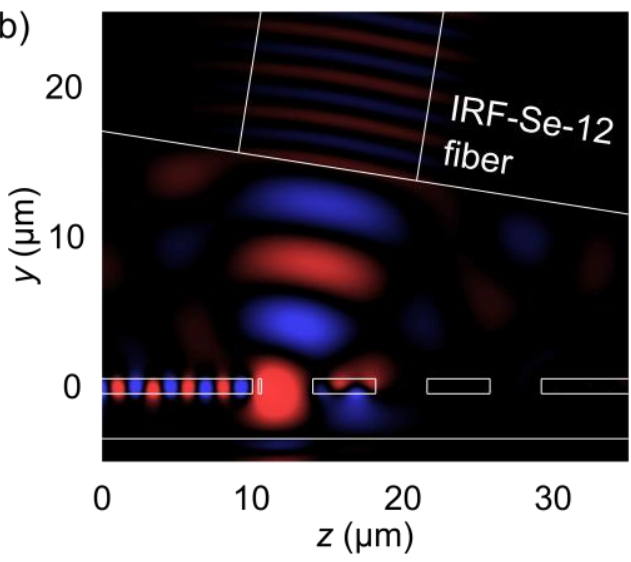

(d)

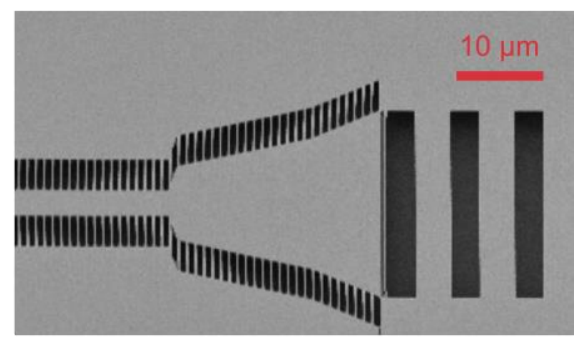

Figure 4. 3D-FDTD simulated coupling efficiency of the suspended germanium micro-antenna as a function of (a) the wavelength and (c) the optical fiber angle. (b) 2D-FDTD electric field propagation for TE polarization at 7.67- $\mu \mathrm{m}$ wavelength. (d) SEM image of a fabricated suspended germanium micro-antenna, including the input waveguide and taper. 


\section{ACKNOWLEDGMENTS}

The authors acknowledge funding from Universidad de Málaga; Ministerio de Ciencia, Innovación y Universidades (MCIU) (PID2019-106747RB-I00); Consejería de Economía, Conocimiento, Empresas y Universidad (CECEU) (UMA18-FEDERJA-219, PY18-1453, PY18-793); and National Research Council of Canada (NRC) Collaborative Science, Technology and Innovation Program (CSTIP) (HTSN 209).

\section{REFERENCES}

[1] Cheben, P., Xu, D.-X., Janz, S.., Densmore, A., "Subwavelength waveguide grating for mode conversion and light coupling in integrated optics," Opt. Express 14(11), 4695-4702 (2006).

[2] Schmid, J. H., Cheben, P., Janz, S., Lapointe, J., Post, E.., Xu, D.-X., "Gradient-index antireflective subwavelength structures for planar waveguide facets," Opt. Lett. 32(13), 1794-1796 (2007).

[3] Schmid, J. H., Cheben, P., Janz, S., Lapointe, J., Post, E., Delâge, A., Densmore, A., Lamontagne, B., Waldron, P., et al., "Subwavelength grating structures in silicon-on-insulator waveguides," Adv. Opt. Technol. 2008, 1-8 (2008).

[4] Bock, P. J., Cheben, P., Schmid, J. H., Delâge, A., Xu, D.-X., Janz, S.., Hall, T. J., "Sub-wavelength grating mode transformers in silicon slab waveguides," Opt. Express 17(21), 19120-19133 (2009).

[5] Schmid, J. H., Cheben, P., Bock, P. J., Halir, R., Lapointe, J., Janz, S., Delage, A., Densmore, A., Fedeli, J.M., et al., "Refractive index engineering with subwavelength gratings in silicon microphotonic waveguides," IEEE Photonics J. 3(3), 597-607 (2011).

[6] Cheben, P., Schmid, J. H., Xu, D.-X., Densmore, A.., Janz, S., "Composite subwavelength-structured waveguide in optical systems," 8,503,839, U.S. Patent (2013).

[7] Cheben, P., Janz, S., Xu, D.-X., Schmid, J. H., Densmore, A.., Lapointe, J., "Interface device for performing mode transformation in optical waveguides," 7,680,371, U.S. Patent (2010).

[8] Cheben, P., Halir, R., Schmid, J. H., Atwater, H. A., Smith, D. R., "Subwavelength integrated photonics," Nature 560(7720), 565-572 (2018).

[9] Halir, R., Ortega-Moñux, A., Benedikovic, D., Mashanovich, G. Z., Wangüemert-Pérez, J. G., Schmid, J. H., Molina-Fernández, Í., Cheben, P., "Subwavelength-grating metamaterial structures for silicon photonic devices," Proc. IEEE 106(12), 2144-2157 (2018).

[10] Soref, R. A., Emelett, S. J., Buchwald, W. R., "Silicon waveguided components for the long-wave infrared region," J. Opt. A Pure Appl. Opt. 8(10), 840-848 (2006).

[11] Soref, R., "Mid-infrared photonics in silicon and germanium," Nat. Photonics 4, 495-497, Nature Publishing Group (2010).

[12] Marris-Morini, D., Vakarin, V., Ramirez, J. M., Liu, Q., Ballabio, A., Frigerio, J., Montesinos, M., AlonsoRamos, C., Le Roux, X., et al., "Germanium-based integrated photonics from near- to mid-infrared applications," Nanophotonics 7(11), 1781-1793 (2018).

[13] Zou, Y., Chakravarty, S., Chung, C.-J., Xu, X., Chen, R. T., "Mid-infrared silicon photonic waveguides and devices," Photonics Res. 6(4), 254-276 (2018).

[14] Hu, T., Dong, B., Luo, X., Liow, T.-Y., Song, J., Lee, C., Lo, G.-Q., "Silicon photonic platforms for midinfrared applications," Photonics Res. 5(5), 417-430 (2017).

[15] Soler Penadés, J., Ortega-Moñux, A., Nedeljkovic, M., Wangüemert-Pérez, J. G., Halir, R., Khokhar, A. Z., Alonso-Ramos, C., Qu, Z., Molina-Fernández, I., et al., "Suspended silicon mid-infrared waveguide devices with subwavelength grating metamaterial cladding," Opt. Express 24(20), 22908-22916 (2016).

[16] Soler Penadés, J., Sánchez-Postigo, A., Nedeljkovic, M., Ortega-Moñux, A., Wangüemert-Pérez, J. G., Xu, Y., Halir, R., Qu, Z., Khokhar, A. Z., et al., "Suspended silicon waveguides for long-wave infrared wavelengths," Opt. Lett. 43(4), 795-798 (2018).

[17] Sánchez-Postigo, A., Wangüemert-Pérez, J. G., Soler Penadés, J., Ortega-Moñux, A., Nedeljkovic, M., Halir, R., El Mokhtari Mimun, F., Xu Cheng, Y., Qu, Z., et al., "Mid-infrared suspended waveguide platform and building blocks," IET Optoelectron. 13(2), 55-61 (2019).

[18] Soler Penades, J., Sanchez-Postigo, A., Nedeljkovic, M., Wangüemert-Pérez, J. G., Ortega-Moñux, A., Halir, R., Khokhar, A. Z., Osman, A., Cheben, P., et al., "Suspended germanium waveguide for infrared wavelengths,” 2019 21st Int. Conf. Transparent Opt. Networks, 1-3, IEEE (2019). 
[19] Dietrich, P.-I., Blaicher, M., Reuter, I., Billah, M., Hoose, T., Hofmann, A., Caer, C., Dangel, R., Offrein, B., et al., "In situ 3D nanoprinting of free-form coupling elements for hybrid photonic integration," Nat. Photonics 12(4), 241-247 (2018).

[20] Marchetti, R., Lacava, C., Carroll, L., Gradkowski, K., Minzioni, P., "Coupling strategies for silicon photonics integrated chips," Photonics Res. 7(2), 201-239 (2019).

[21] Tamir, T., Peng, S. T., “Analysis and design of grating couplers,” Appl. Phys. 14(3), 235-254 (1977).

[22] Taillaert, D., Bienstman, P., Baets, R., "Compact efficient broadband grating coupler for silicon-on-insulator waveguides," Opt. Lett. 29(23), 2749-2751 (2004).

[23] Sánchez-Postigo, A., Gonzalo Wangüemert-Pérez, J., Luque-González, J. M., Molina-Fernández, Í., Cheben, P., Alonso-Ramos, C. A., Halir, R., Schmid, J. H., Ortega-Moñux, A., "Broadband fiber-chip zero-order surface grating coupler with 0.4 dB efficiency," Opt. Lett. 41(13), 3013-3016 (2016).

[24] Sacher, W. D., Huang, Y., Ding, L., Taylor, B. J. F., Jayatilleka, H., Lo, G.-Q., Poon, J. K. S., "Wide bandwidth and high coupling efficiency $\mathrm{Si}_{3} \mathrm{~N}_{4}$-on-SOI dual-level grating coupler," Opt. Express 22(9), 1093810947 (2014).

[25] Wade, M. T., Pavanello, F., Kumar, R., Gentry, C. M., Atabaki, A., Ram, R., Stojanovic, V., Popovic, M. A., "75\% efficient wide bandwidth grating couplers in a $45 \mathrm{~nm}$ microelectronics CMOS process," 2015 IEEE Opt. Interconnects Conf. OI 2015 4(c), 46-47 (2015).

[26] Ayata, M., Fedoryshyn, Y., Koch, U., Leuthold, J., "Compact, ultra-broadband plasmonic grating couplers," Opt. Express 27(21), 29719-29729 (2019).

[27] Sánchez-Postigo, A., Ortega-Moñux, A., Pereira-Martín, D., Molina-Fernández, Í., Halir, R., Cheben, P., Soler Penadés, J., Nedeljkovic, M., Mashanovich, G. Z., et al., "Design of a suspended germanium micro-antenna for efficient fiber-chip coupling in the long-wavelength mid-infrared range," Opt. Express 27(16), 2230222315 (2019).

[28] Joannopoulos, J. D., Johnson, S., Winn, J. N., Meade, R. D., Photonic Crystals: Molding the Flow of Light, Princet. Univ. Press, Princeton University Press (2011).

[29] "SMF-28 ${ }^{\circledR} ",<$ https://www.corning.com/worldwide/en/products/communication-networks/products/fiber/smf28-ultra.html> (8 February 2021).

[30] "Infrared Fiber Optics | IRFlex Corporation.”, <https://www.irflex.com/products/irf-se-series/> (8 February 2021).

[31] Zavargo-Peche, L., Ortega-Moñux, A., Wangüemert-Pérez, J. G., Molina-Fernández, Í., "Fourier based combined techniques to design novel sub-wavelength optical integrated devices," Prog. Electromagn. Res. 123(January), 447-465 (2012). 